\title{
Predicción de variables operacionales en el proceso de Producción de Crudos por Segregación Gravitacional Asistida por Vapor (SAGD)
}

\author{
(Prediction of operational variables involved in the Production \\ Process of Crude Oil by Steam Assisted Gravity Drainage \\ (SAGD) )
}

\author{
David Lowy ${ }^{1}$, Geovanna Garrido ${ }^{1}$, Vinicio Melo ${ }^{1}$
}

\begin{abstract}
Resumen:
Este trabajo presenta las variables de yacimiento y operacionales que influyen en la implementación del proceso de Segregación Gravitacional Asistida por Vapor (SAGD) para la Producción de Crudos Pesados y Extra-pesados. Este proceso consiste en la perforación de dos pozos horizontales paralelos, uno por encima del otro, donde el pozo superior es usado para inyectar vapor y el pozo inferior es usado como productor. La eficiencia del proceso se ve altamente afectada por los parámetros del yacimiento y por los parámetros operacionales (espaciamiento vertical de los pozos, presión de inyección, período de precalentamiento, entre otros). Además, se determinó de manera teórica la predicción de la tasa máxima de extracción de petróleo, mediante un ejemplo aplicativo utilizando datos reales que se disponen actualmente del Campo Pungarayacu - Bloque 20, a nivel de yacimiento.
\end{abstract}

Palabras clave: Segregación Gravitacional Asistida por Vapor (SAGD); cámara de vapor; crudos pesados y extra-pesados; tasa máxima de extracción; vapor de agua.

\begin{abstract}
:
This paper presents the oil deposit and operational variables that influence the implementation of the Steam Assisted Gravity Drainage (SAGD) process for the Production of Heavy and Extraheavy Crude Oil. This process consists of drilling two parallel horizontal wells, one above the other, where the upper well is used for steam injection and the lower well is used for production. The efficiency of the process is greatly affected by the deposit and operating parameters (vertical spacing of wells, injection pressure, preheating period, among others). Furthermore, the prediction of the maximum rate of oil extraction was determined using an example with currently available real data of Block 20 of the Pungarayacu Field.
\end{abstract}

Keywords: Steam Assisted Gravity Drainage (SAGD); steam chamber; heavy and extra-heavy crude oil; maximum rate of extraction; steam water.

\section{Introducción}

En la actualidad, la cantidad de descubrimientos de acumulaciones convencionales de petróleo se están haciendo cada vez menores, por tal razón la atención de la industria petrolera en muchos lugares del mundo se está desplazando hacia la explotación de petróleo pesado $\left(10^{\circ} \mathrm{API}-\right.$ $\left.22.3^{\circ} \mathrm{API}\right)$, ya que junto con el petróleo extra-pesado $\left(<10^{\circ} \mathrm{API}\right)$ y el bitumen conforman aproximadamente un $70 \%$ de los recursos de petróleo totales del mundo. Las acumulaciones de

\footnotetext{
${ }^{1}$ Universidad Tecnológica Equinoccial, Quito - Ecuador (\{lada38656, grge96059, vinicio.melo \}@ute.edu.ec)
} 
crudos pesados y extra-pesados han adquirido bastante importancia en las últimas décadas, ya que las compañías operadoras de petróleo han tenido que acudir a la explotación de yacimientos no convencionales de petróleo, para completar la producción que se demanda mundialmente.

El petróleo pesado promete desempeñar un rol muy importante en el futuro de la industria petrolera y muchos países están tendiendo a incrementar su producción, revisar las estimaciones de reservas, invertir en infraestructura y comprobar nuevas tecnologías debido a que estas acumulaciones requieren el uso de tecnologías especiales de explotación, de modo de hacerlas económicamente rentables. Entre estas nuevas tecnologías se encuentra el proceso de Segregación Gravitacional Asistida por Vapor (SAGD, sus siglas en Inglés: Steam Assisted Gravity Drainage).

El presente trabajo resulta de fundamental importancia en el área de la Ingeniería de Yacimientos y en los métodos de recuperación térmica de petróleo; de igual manera, implica un aporte técnico al tema de los parámetros de yacimiento y operacionales que afectan en mayor medida al éxito y rendimiento de un proceso SAGD.

Además, se determinó teóricamente una predicción de la tasa máxima de extracción que se alcanza en un proceso SAGD de crudos pesados y extra-pesados, utilizando datos del Campo Pungarayacu (Bloque 20), a nivel de yacimiento.

El Campo Pungarayacu está localizado en la provincia de Napo, en el flanco Este de la Cordillera Oriental. El material útil que se halla conformando el yacimiento es la arenisca bituminosa de la Formación Hollín que se presenta en Hollín Superior, Medio e Inferior. El yacimiento se encuentra en la Formación Hollín, a profundidades que van de 0 a 700 metros desde la superficie, variando de Norte a Sureste con una relación directa entre la topografía, y el espesor de la sobrecarga destacándose en este reservorio tres cuerpos de areniscas.

Las arenas de la Formación Hollín Inferior constituyen el cuerpo más importante del reservorio, con un espesor que varía entre 23 y 57 metros. Según información de la Secretaría Nacional de Hidrocarburos del 2008, esta arenisca es de color gris claro, grano fino a medio, forma subangular, de selección regular a buena, porosidad igual a $22.4 \%$, saturación de agua de $45 \%$, entre otras características litológicas y petrofísicas.

Las arenas del miembro medio de la formación están separadas del miembro inferior por una capa impermeable de limolitas y arcillolitas, su espesor varía de 21 a 42 metros, con cambios de permeabilidad y porosidad, tanto en sentido vertical como en sentido horizontal. En la Formación Hollín Superior las arenas están separadas en su parte inferior por una capa de lutitas y en la parte superior por calizas o lutitas calcáreas pertenecientes a la Formación Napo, el espesor de arena varía de 3 a 12 metros. 
Dentro de los estudios realizados al yacimiento, se ha determinado sus reservas técnicas existentes, en base a los datos recopilados para saber cuán posible será la producción de este campo, ya que se trata de crudo pesado.

Según información presentada por la empresa IVANHOE ${ }^{2}$ en junio de 2008, el Campo Pungarayacu contendría 4.500 millones de barriles in situ, con un factor de recobro utilizando nuevas tecnologías entre el $35 \%$ al $45 \%$ y con una recuperación adicional aproximada del $14 \%$ del petróleo original en sitio.

Los valores para la descripción físico - química del petróleo del Campo Pungarayacu, fueron tomados de análisis realizados a muestras recogidas del talud norte de la carretera Hollín-LoretoCoca, cerca del kilómetro 3.5 en el margen oriental del río Chontayacu. Estos valores se los presenta en las Tablas 1 y 2.

Tabla 1. Resultados de análisis de muestra de petróleo aflorante (petróleo en superficie).

\begin{tabular}{|l|c|}
\hline \multicolumn{1}{|c|}{ Propiedad y unidad } & Valores \\
\hline Viscosidad (m.Pa.s) a $40^{\circ} \mathrm{C}$ & 500000 \\
\hline Viscosidad (m.Pa.s) a $60^{\circ} \mathrm{C}$ & 144000 \\
\hline Viscosidad (m.Pa.s) a $88^{\circ} \mathrm{C}$ & 7270 \\
\hline${ }^{\circ} \mathrm{API}$ & 6.6 \\
\hline Densidad (g/cm ${ }^{3}$ ) a $50^{\circ} \mathrm{C}$ & 1.002 \\
\hline $\begin{array}{l}\text { Contenido de azufre }(\% \text { en } \\
\text { peso) }\end{array}$ & 3.4 \\
\hline Petróleo residual & 69.1 \\
\hline
\end{tabular}

Fuente. (Secretaría Nacional de Hidrocarburos, 2008).

\section{Proceso SAGD}

\section{Fundamentación del proceso SAGD}

El Proceso de Segregación Gravitacional Asistida por Vapor es un método sistemático de inyección continua de vapor, que permite incrementar el recobro de petróleo en yacimientos de crudos pesados y extra-pesados. El arreglo de los pozos, consiste en dos pozos horizontales paralelos perforados cerca de la base del yacimiento, de los cuales uno es el inyector de vapor y está ubicado encima de un pozo productor (ver Figura 1). Su principal mecanismo de empuje es causado por la fuerza de gravedad, la cual produce el movimiento del crudo hacia un pozo productor.

\footnotetext{
${ }^{2}$ Informe del estudio de impacto ambiental para el desarrollo de la fase de prospección geofísica del Bloque 20, IVANHOE Energy Ecuador Inc.
} 
Ambos pozos deben estar alineados horizontalmente y verticalmente separados por una corta distancia. Antes de iniciar el proceso, los pozos deben ser calentados previamente mediante la circulación de vapor a lo largo de la longitud total de los pozos (a través de la tubería de producción), con la finalidad de crear una comunicación térmica entre los mismos.

Tabla 2. Características Físico-Químicas del Campo Pungarayacu.

\begin{tabular}{|c|c|c|}
\hline Características & Unidad & Resultado \\
\hline Peso específico a $15.5^{\circ} \mathrm{C}$ & $\mathrm{g} / \mathrm{cm}^{3}$ & 1.07 \\
\hline Densidad & ${ }^{\circ} \mathrm{API}$ & 5.5 \\
\hline Densidad Relativa & $60 / 60$ & 1.0328 \\
\hline Viscosidad a $38.7^{\circ} \mathrm{C}$ & SSF & 24163 \\
\hline Viscosidad a $50^{\circ} \mathrm{C}$ & SSF & 11474 \\
\hline Viscosidad a $98.7^{\circ} \mathrm{C}$ & SSF & 900 \\
\hline Viscosidad a $134^{\circ} \mathrm{C}$ & SSF & 172 \\
\hline Punto de Inflamación & ${ }^{\circ} \mathrm{C}$ & 170.7 \\
\hline Punto de Combustión & ${ }^{\circ} \mathrm{C}$ & 179.3 \\
\hline Calor Específico & $\mathrm{kcal} / \mathrm{kg}$ & 9887.4 \\
\hline Contenido de Aromáticos & $\%$ & 40.79 \\
\hline Contenido de Naftenos & $\%$ & 32.50 \\
\hline Contenido de Parafinas & $\%$ & 26.71 \\
\hline Contenido de Azufre & $\%$ en peso & 3.29 \\
\hline Vanadio & ppm & 38.30 \\
\hline Níquel & ppm & 243.20 \\
\hline Sodio & ppm & 92.30 \\
\hline Hierro & ppm & 156.60 \\
\hline Calcio & ppm & 43.10 \\
\hline Potasio & ppm & 59.00 \\
\hline Magnesio & ppm & 41.40 \\
\hline Contenido de Petróleo & $\%$ en peso & 10 \\
\hline Contenido de Sedimentos & $\%$ en peso & 61.0 \\
\hline Humedad & $\%$ en volumen & 0.23 \\
\hline Penetración & $\mathrm{l} / 10 \mathrm{~mm}$ & 45 \\
\hline Punto de Ablandamiento & ${ }^{\circ} \mathrm{C}$ & 42.2 \\
\hline Ductibilidad & $\mathrm{cm}$ & 35 \\
\hline Solubilidad en CC14 & $\%$ en peso & 88.3 \\
\hline Factor de Caracterización & KUOP & 10.67 \\
\hline
\end{tabular}

Fuente. (Secretaría Nacional de Hidrocarburos, 2008).

Posteriormente, se adiciona la inyección continua de vapor por el anular del pozo inyector, sobre el cual se forma una zona de vapor saturado denominada "Cámara de Vapor". Durante el 
desarrollo de la cámara de vapor dentro del yacimiento, se presentan tres etapas o períodos, que se originan sucesivamente, a medida que ésta se forma. Estos períodos son:

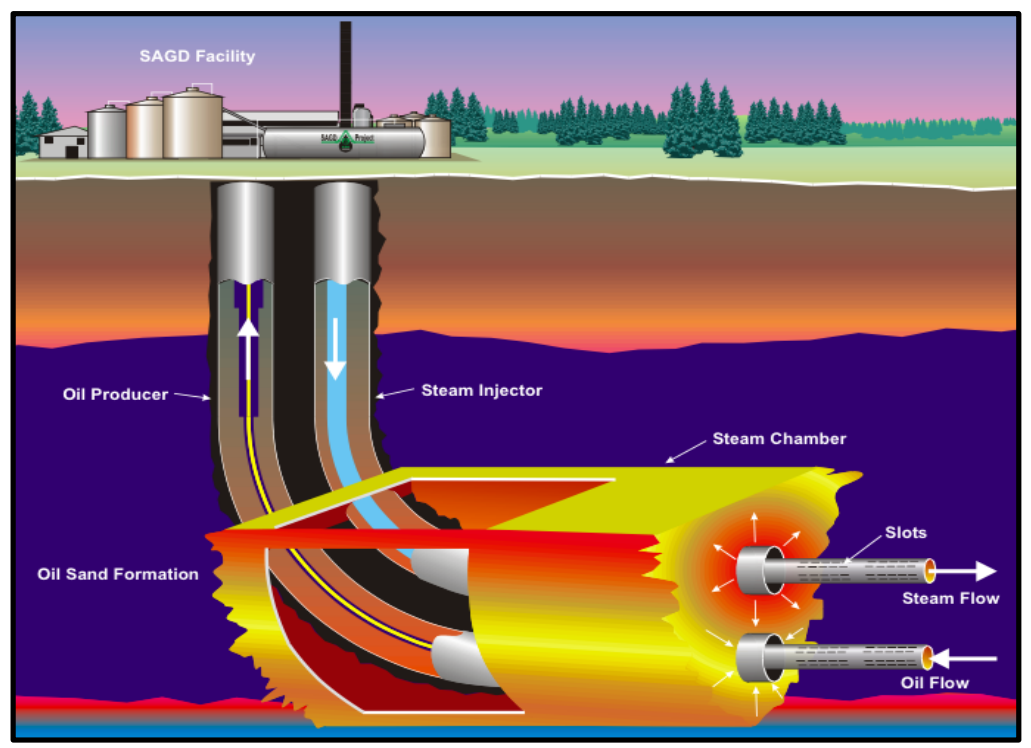

Figura 1. Esquema Proceso SAGD con pozos horizontales. Fuente. (Alvarado, 2002).

\section{- Período de Crecimiento Vertical de la Cámara.}

Es conocida como período de ascenso de la cámara o período de incremento de potencial, y ocurre desde el momento en que comienza la inyección del vapor en el yacimiento hasta que éste llega al tope del mismo. En esta etapa, la tasa de producción aumenta progresivamente.

\section{- Período de Expansión Lateral.}

Se caracteriza por una tasa de producción que se mantiene estable. Esta etapa se inicia desde el momento en que la cámara llega al tope del yacimiento y comienza a expandirse solamente hacia los lados, hasta abarcar la longitud horizontal del pozo productor.

\section{- Período de Declinación.}

Es la última fase en la cual la tasa de producción comienza a disminuir. La etapa se inicia desde el momento en que la cámara de vapor llega al final de la longitud horizontal del pozo productor y finaliza cuando la altura del volumen de crudo entre los pozos (ubicada de los límites del área de drenaje de la cámara) comienza a decrecer con lo que disminuye igualmente la tasa de drenaje, hasta convertirse en un proceso no económico. Al llegar a este punto se concluye que el proyecto ha finalizado.

La presión en la cámara de vapor normalmente se mantiene constante durante todo el proceso, y se encuentra rodeada de arena petrolífera fría. El vapor fluye a través de la arena dentro de la cámara hasta alcanzar la interfase "Arena Petrolífera - Cámara de Vapor", donde se condensa y el calor liberado se transmite por conducción a la arena petrolífera fría. 
De esta manera, es calentado el petróleo que se encuentra cerca de la superficie de condensación, permitiendo a su vez que tanto el petróleo como los condensados sean drenados por gravedad hacia el pozo productor.

Durante este proceso, el petróleo se mueve en dirección contraria a la de avance de la cámara de vapor. El petróleo y los condensados drenan hacia abajo y el vapor hacia arriba, ya que el vapor tiende a subir, y tanto los condensados y el crudo caliente tienden a caer al fondo (por ser más pesados). Por lo tanto, el flujo es causado por la fuerza de gravedad (Figura 2).

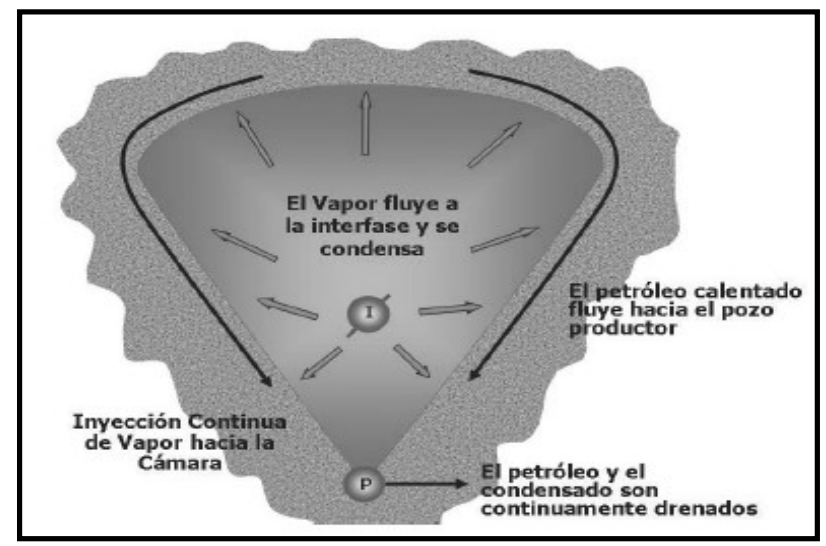

Figura 2. Cámara de vapor del Proceso SAGD.

Fuente. (Montes, 2012).

En general, el mecanismo del proceso SAGD, se puede resumir en cuatro pasos: el vapor se condensa en la interfase; el petróleo y el condensado son drenados hacia el pozo productor; el flujo es causado por la gravedad y crecimiento de la cámara de vapor hacia arriba y hacia los lados.

La producción de los pozos se realiza mediante levantamiento natural de los fluidos. Debido a que las temperaturas de operación son generalmente mayores a $200^{\circ} \mathrm{C}$, para tales temperaturas no existen sistemas de bombeo confiables que puedan manejar la cantidad de producción esperada. Por consiguiente, el tipo de completación es totalmente atípica y única bajo este tipo de tecnología. Adicionalmente de utilizar el mecanismo de producción de levantamiento de flujo natural con vapor, este puede ser apoyado, mediante levantamiento con inyección de gas.

\section{Criterios de diseño del proceso SAGD}

Los criterios de selección sugieren las bases para llevar a cabo un proyecto rentable bajo la tecnología SAGD, los cuales están basados en resultados de simulaciones. Analizar y evaluar la influencia de la arquitectura geológica y las características de yacimientos, determina la factibilidad del proceso en yacimientos de crudos pesados y extrapesados.

La mayoría de las características geológicas de la formación definen la forma y el tamaño de la cámara de vapor, así como la viabilidad del proceso. Dentro de los aspectos geológicos que 
influyen, constan: espesor de la arena, capa impermeable, acuíferos, capas de gas, heterogeneidades de la formación y el efecto del buzamiento de la formación.

En cuanto, a las consideraciones del yacimiento, se requiere de una alta relación crudo - vapor (SOR), debido a que toda la región del yacimiento depletado requiere ser calentado a la temperatura del vapor. Según esto, es necesario contar con buenas propiedades petrofísicas, tales como alta porosidad $(\Phi)$, alta permeabilidad $(k)$ y alta saturación de crudo (So). Las formaciones con baja viscosidad $(\mu)$, están asociadas con yacimientos de mayores profundidades, los cuales no son atractivos para el proceso SAGD.

Cualquier yacimiento en particular, debe ser analizado y simulado con sus características originales, a fin de asegurar la viabilidad del proceso, sin embargo, los factores de diseño deseables para un proceso SAGD son:

- Arenas continuas con espesores mayores a 15 metros (49 pies) para viscosidades de hasta $10000 \mathrm{cp}$. Para crudos menos viscosos se pueden aceptar espesores de hasta 10 metros (33 pies).

- Permeabilidad mayor a 1 Darcy.

- Alta saturación de crudo (mayor al $80 \%$ ).

- Alta porosidad (mayor al 30\%).

- Gravedad API menor de $20^{\circ}$.

- Presión actual del yacimiento menor de 1000 psi.

- Profundidad menor a 1372 metros (4500 pies).

- Evitar la presencia de capas de gas y acuíferos (tanto en el tope como la base del yacimiento).

- No deben existir barreras lutíticas de gran continuidad o extensión y poco espesor (menores a 2 metros).

- Presencia de una capa impermeable en el tope de la formación.

- Contenido de arcillas hinchables dentro de la formación menor a $10 \%$.

- Evitar la presencia de fallas y fracturas.

Además, existen dos formas generales de configuración de los pozos para desarrollar el proceso SAGD (ver Figura 3) :

- Pozo inyector horizontal y pozo horizontal productor, los pozos deben estar configurados de forma paralela (inyector ubicado encima del productor) y alineados en la misma dirección.

- Pozo inyector vertical y pozo horizontal productor, donde su ejecución es más económica y sencilla de construir. 

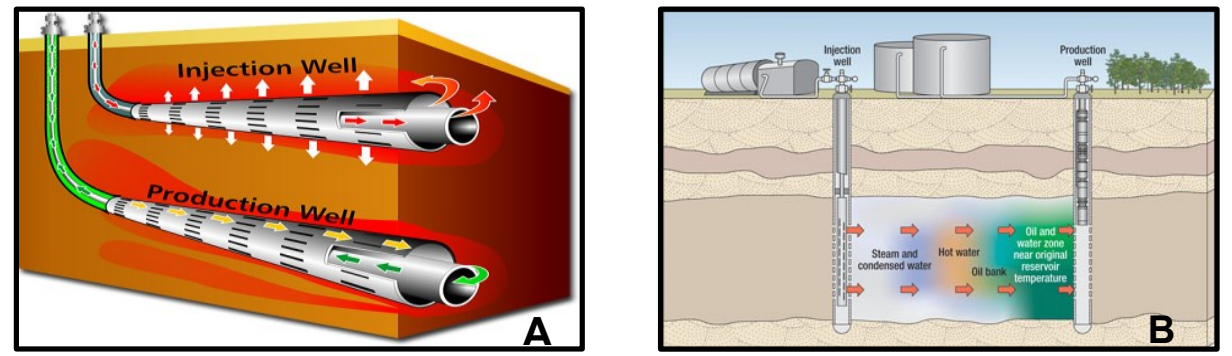

Figura 3. A. Pozo inyector horizontal posicionado encima de un pozo horizontal productor B. Pozo inyector vertical posicionado encima de un pozo horizontal productor.

Fuente. (IVANHOE, 2013).

\section{Factores que determinan la efectividad del proceso SAGD}

Existen diversos aspectos a considerar para un correcto y óptimo desarrollo de la tecnología SAGD, entre estos tenemos:

\section{- Distancia de separación de pozos}

La separación vertical como la alineación entre los pozos, debe ser lo más precisa posible. Los pozos son espaciados entre 4 a 10 metros de separación, distancia típica (a nivel mundial) para crudos pesados y está basada en simulaciones. Además, esta variable depende de la viscosidad del crudo; para crudos muy viscosos, el pozo inyector es ubicado generalmente a 5 metros de separación del pozo productor. Si el crudo del yacimiento es menos viscoso, el pozo inyector puede ser ubicado en una sección superior del yacimiento, permitiendo una mayor separación entre ambos pozos.

\section{- Espaciamiento de parejas de pozos}

Para un mejor recobro del yacimiento y eficiencia del proceso, siempre es recomendable, iniciar un proyecto SAGD con al menos dos pares de pozos, los cuales deben estar espaciados a cierta distancia. El espaciamiento entre las parejas de pozos oscila entre 100 a 150 metros; sin embargo, el espaciamiento óptimo dependerá de los resultados de simulaciones. Mientras mayor sea el espaciamiento entre las parejas de pozos, resultará en mayores tasas de producción acumuladas por cada pareja de pozos.

\section{- Instrumentación de la completación}

La instrumentación en la completación de los pozos, es necesaria para monitorear y evaluar el crecimiento progresivo de la cámara de vapor a fin de optimizar su desarrollo durante la vida operativa de la pareja de pozos y lograr un drenaje eficiente del yacimiento. Puede ser supervisado con la instalación de sensores de presión (capilares), sensores de temperatura (termocuplas) y mediante sistemas de distribución de temperaturas de fibra óptica instalados en el fondo de los pozos. En cuanto al control del proceso, se puede inferir de los patrones observados en el comportamiento del mismo, a través de las mediciones directas del fondo del pozo. 
Las mediciones de presión y temperatura pueden indicar una referencia de la evolución del proceso de transferencia de calor en el yacimiento y del grado de desplazamiento de vapor a lo largo de la completación hacia el yacimiento. Las medidas de temperatura permiten detectar ciertas condiciones, tales como, el predominio del vapor, zonas ladronas de vapor, zonas o capas con alta permeabilidad, los cuales proporcionan información para estudios posteriores.

Uno de los factores claves del proceso SAGD, es la condición o grado de subenfriamiento, el cual debe ser controlado para evitar la conificación del vapor y contribuir a reducir la disipación del calor. La condición de subenfriamiento es una función de la presión y temperatura del pozo productor, que influyen en el mantenimiento y desarrollo de la cámara de vapor.

En cuanto a los parámetros operacionales, los que resultan más influyentes en el proceso son: la tasa de inyección de vapor, la duración del período de precalentamiento y la calidad del vapor inyectado.

\section{- Inyección de vapor}

El procedimiento para la inyección de vapor se inicia con la circulación de vapor dentro de ambos pozos, inyector y productor, para calentar el crudo por conducción térmica, de forma de iniciar la comunicación vertical entre ambos pozos.

La tasa de inyección de vapor es de vital importancia para el desarrollo de la cámara de vapor y por ende, para el éxito del proceso SAGD. El desempeño de la cámara y de la producción del pozo será mejor mientras la relación o tasa acumulada "liquido (crudo + agua)/vapor", sea más alta.

La calidad del vapor inyectado influye directamente en la cantidad de energía transmitida al yacimiento. De esta forma, es necesario que el vapor inyectado llegue al yacimiento con la calidad máxima que las condiciones operacionales permitan. La cantidad de calor que el vapor de agua es capaz de transmitir al yacimiento va a variar en función de su calidad: a mayor calidad mayor entalpía, y por ende, mayor calor inyectado al yacimiento.

\section{- Período de precalentamiento}

Es necesario que se lleve a cabo el período de calentamiento del yacimiento, 1 a 2 meses, antes del inicio de las operaciones de inyección y producción, con el fin de calentar el crudo y aumentar su movilidad inicial, puesto que este procedimiento es favorable en el rendimiento general del proceso SAGD. 


\section{Tasa máxima de producción de petróleo}

La ecuación de la tasa máxima de producción de petróleo de un campo que está produciendo bajo el proceso SAGD, preestablecida en forma teórica.

La tasa a la cual el crudo se drena durante el esparcimiento de la cámara de vapor, sin que se produzca simultáneamente la producción de vapor en el pozo productor es:

$$
\mathrm{q}=2 \mathrm{~L} \sqrt{\frac{1.3 \mathrm{~kg} \phi \Delta \mathrm{S}_{\mathrm{o}} \mathrm{h}}{\mathrm{m} v_{\mathrm{S}}}}
$$

Esta ecuación ha sido llamada la ecuación del "drenaje lineal” o ecuación "Lindrain” 3.

El procedimiento de cálculo para determinar la tasa empírica máxima de producción de petróleo para un campo determinado es:

a) Determinar la temperatura del vapor con el valor de la presión en la cámara de vapor. Esto se puede hacer usando las tablas de vapor o, como los datos están en el sistema internacional de unidades SI, podemos utilizar usando la correlación de Stanford-Moss que es la siguiente:

$$
T_{S}=\frac{326.57}{\sqrt{9.8809-\log P_{S}}-2.4223}-273.15
$$

b) Obtener la viscosidad cinemática en función de la temperatura usando la ecuación de Walther aplicada a crudos de Canadá, Estados Unidos y Venezuela, modificada por Svrcek y Mehrotra en 1988, donde sólo se requiere la viscosidad en un solo punto, en el cual se conozca también la densidad.

La correlación es la siguiente:

$$
\log [\log (v+0.7)]=m \log (T+273)+b
$$

Donde $b$ es una constante para un petróleo en particular y $m$ es igual a:

$$
m=0.3249-0.4106 b
$$

Con los valores de $b$ y $m$ obtenidos, reemplazarlos en la siguiente ecuación a la temperatura de saturación del vapor obtenida en el paso 1:

$$
v=10^{10^{[b+m \log (T+273)]}}-0.7
$$

\footnotetext{
${ }^{3}$ Para un desglose más detallado de la deducción de esta fórmula, remítase a Alvarado \& Banzer (2002).
} 
c) Calcular la siguiente integral definida para encontrar el valor de $m$ determinado en $\mathrm{s} / \mathrm{m}^{2}$ :

$$
\frac{1}{m v_{S}}=\int_{T_{R+\delta}}^{T_{S}} \frac{d T}{v\left(T-T_{R}\right)}
$$

Para esto, al integrando se lo grafica contra la temperatura hasta el valor de temperatura de saturación del vapor $T_{S}$.

El área bajo la curva de dicho gráfico es proporcional al flujo en ese rango de temperatura. Se debe calcular el valor exacto del área bajo la curva para determinar el parámetro adimensional $\mathrm{m}$.

Se calcula la tasa máxima de producción para el proceso SAGD utilizando la ecuación empírica denominada ecuación de drenaje lineal para los dos lados de la cámara, previamente antes definida.

$$
\mathrm{q}=2 \mathrm{~L} \sqrt{\frac{1.3 \mathrm{~kg} \phi \Delta \mathrm{S}_{\mathrm{o}} \mathrm{h}}{\mathrm{m} v_{\mathrm{S}}}}
$$

El valor de $L$ representa la longitud del pozo horizontal, que de acuerdo a experiencias de campo y de simulación matemática en Canadá, utilizando crudos de similares características petrofísicas, físicas y químicas del crudo del Campo Pungarayacu, se sugiere que la longitud del pozo horizontal sea de 400 metros, y considerando que los pozos horizontales se ubiquen lo más cerca posible a la base de la formación, para minimizar las pérdidas por crudo no recuperable.

El área de drenaje se determina en el período de declinación de la cámara de vapor. Esta etapa se inicia desde el momento en que la cámara de vapor llega al final de la longitud horizontal del pozo productor, utilizándose el valor de 400 metros ya mencionado, y finaliza cuando la altura del volumen de crudo entre los pozos (ubicada en los límites del área de drenaje de la cámara, que de acuerdo a experiencias de campo como de simulación se ha determinado que está entre 30 y 40 metros) comienza a decrecer, con lo que disminuye igualmente la tasa de drenaje hasta convertirse en un proceso no económico. Por lo que el área de drenaje se encuentra aproximadamente entre 12000 a $16000 \mathrm{~m}^{2}$.

Antes de emplear la ecuación propuesta, se debe determinar los valores de los parámetros que intervienen en dicha ecuación y convertirlos a un sistema de unidades consistente. 


\section{Resultados y Discusión}

A continuación se presenta el procedimiento de cálculo para estimar la tasa máxima de producción que se podría esperar para un pozo horizontal de $400 \mathrm{~m}$, perforado en la base del yacimiento, en un proyecto SAGD en el Campo Pungarayacu, cuyo petróleo tiene una viscosidad cinemática de $127.7 \mathrm{cSt}$ a $100^{\circ} \mathrm{C}$, con una presión en la cámara de vapor de $1.9 \mathrm{MPa}$ (275 lpca) y con los siguientes parámetros mostrados en la Tabla 3 :

Tabla 3. Datos del Campo Pungarayacu.

\begin{tabular}{|l|c|c|}
\hline \multicolumn{1}{|c|}{ Variables } & Valores & Unidades \\
\hline Altura de cámara vapor $\mathrm{h}$ & 30 & metros \\
\hline Saturación de petróleo So & 0.75 & fracción \\
\hline Densidad petróleo a $100^{\circ} \mathrm{C}$ & 940 & $\mathrm{~kg} / \mathrm{m}^{3}$ \\
\hline Petróleo residual Sor & 0.15 & fracción \\
\hline Difusividad térmica $\alpha$ & 0.05 & $\frac{\mathrm{m}^{2}}{\text { día }}$ \\
\hline Temperatura inicial $T_{R}$ & 14 & ${ }^{\circ} \mathrm{C}$ \\
\hline Permeabilidad K & 1.2 & Darcy \\
\hline Porosidad $\phi$ & 0.224 & fracción \\
\hline
\end{tabular}

Fuente. (Secretaría Nacional de Hidrocarburos, 2008)

\section{- Procedimiento de cálculo}

a) Determinar la temperatura del vapor con el valor de la presión en la cámara de vapor que es 1.9 MPa (275 Ipca), usando la correlación de Stanford-Moss:

$$
\begin{gathered}
T_{S}=\frac{326.57}{\sqrt{9.8809-\log P_{S}}-2.4223}-273.15 \\
T_{S}=\frac{326.57}{\sqrt{9.8809-\log (1.9)}-2.4223}-273.15 \\
\mathrm{~T}_{\mathrm{S}}=210^{\circ} \mathrm{C}
\end{gathered}
$$

b) Obtener la viscosidad cinemática en función de la temperatura, mediante la correlación de Svrcek y Mehrotra:

$$
\log [\log (v+0.7)]=m \log (T+273)+b
$$

Aplicando la correlación obtenemos para $100^{\circ} \mathrm{C}$ :

$$
v=\frac{\mu}{\rho}=127.7
$$




$$
\begin{gathered}
\log [\log (127.7+0.7)]=m \log (100+273)+b \\
0.323986998=2.571708832 \mathrm{~m}+\mathrm{b}
\end{gathered}
$$

Donde $b$ es una constante para un petróleo en particular y $m$ es igual a:

$$
\mathrm{m}=0.3249-0.4106 \mathrm{~b}
$$

Reemplazando el valor de $m$ en la ecuación, obtenemos el valor de $b$ :

$$
\begin{gathered}
0.323986998=2.571708832(0.3249-0.4106 \mathrm{~b})+\mathrm{b} \\
0.323986998=0.835548199-1.055943646 \mathrm{~b}+\mathrm{b} \\
-0.511561201=-0.055943646 \mathrm{~b} \\
\mathrm{~b}=9.144223474
\end{gathered}
$$

En la ecuación de $m$, se reemplaza el valor obtenido de $b$

$$
\begin{gathered}
m=0.3249-0.4106 b \\
\mathrm{~m}=0.3249-0.4106(9.144223474)=-3.429718158
\end{gathered}
$$

Con los valores de b y m obtenidos, reemplazarlos en la siguiente ecuación a la temperatura de saturación del vapor obtenida en el paso 1:

$$
\begin{gathered}
v=10^{10^{[b+m \log (T+273)]}}-0.7 \\
v=10^{10^{[9.1442234 t 3.429711818 \operatorname{lsg}(210+273)]}}-0.7 \\
v_{\mathrm{s} @ 210^{\circ} \mathrm{C}}=6.74 \mathrm{cSt}
\end{gathered}
$$

c) Se calcula la siguiente integral definida para encontrar el valor de $m$ determinado en $\mathrm{s} / \mathrm{m}^{2}$ :

$$
\frac{1}{m v_{S}}=\int_{T_{R+\delta}}^{T_{S}} \frac{d T}{v\left(T-T_{R}\right)}
$$

Para esto, al integrando se lo grafica contra la temperatura hasta el valor de temperatura de saturación del vapor $T_{s}$. La siguiente Figura 4 nos muestra este gráfico. 


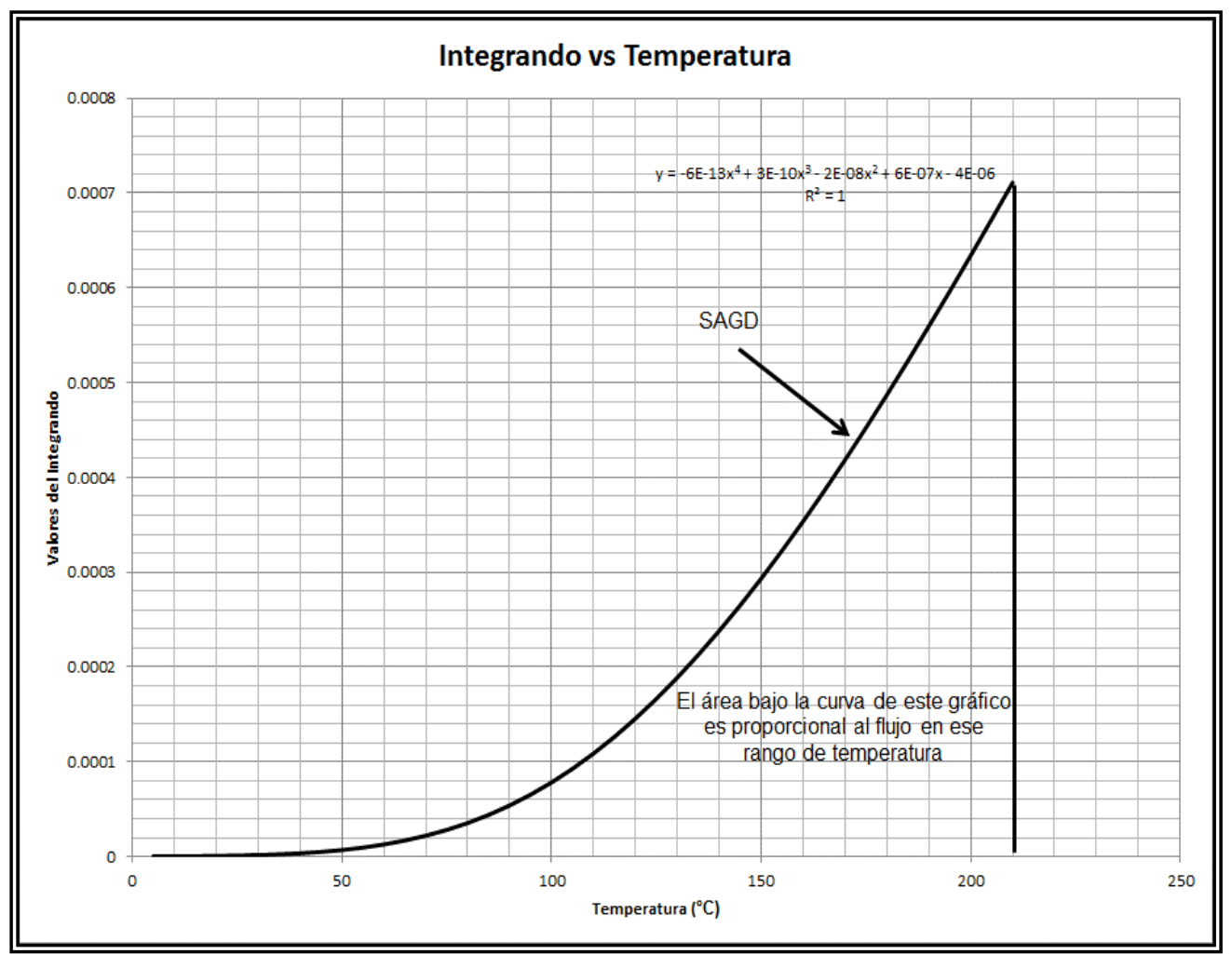

Figura 4. Gráfico del Integrando vs. Temperatura.

Se ignora el área cercana a la temperatura inicial del reservorio $T_{R}$. Con Excel, agregar la línea de tendencia del gráfico para determinar la ecuación de la curva.

Una ecuación polinómica de cuarto grado es la que da un ajuste exacto de la curva $\left(R^{2}=1\right)$, dicha ecuación es:

$$
y=-6 \times 10^{-13} x^{4}+3 \times 10^{-10} x^{3}-2 \times 10^{-8} x^{2}+6 \times 10^{-7} x-4 \times 10^{-6}
$$

De esta ecuación, se tiene que determinar el valor exacto de su integral definida para el intervalo entre $0^{\circ} \mathrm{C}$ a $210^{\circ} \mathrm{C}$ con respecto a la variable $x$, esto se hace para encontrar el área bajo la curva de la ecuación. Esto se lo puede hacer automáticamente en una calculadora de integrales:

$$
\int_{0^{\circ} C}^{210^{\circ} C}\left(-6 \times 10^{-13} x^{4}+3 \times 10^{-10} x^{3}-2 \times 10^{-8} x^{2}+6 \times 10^{-7} x-4 \times 10^{-6}\right) d x=0.047501538
$$

Por lo tanto:

$$
\frac{1}{\mathrm{~m} v_{\mathrm{S}}}=0.047501538
$$

En el paso 2, se calculó previamente el valor de la viscosidad cinemática a $210^{\circ} \mathrm{C}$ y con ello calcular el valor del parámetro $m$. 


$$
\begin{aligned}
& \frac{1}{\mathrm{~m}(6.7)}=0.047501538 \\
& 1=0,3182603046 \mathrm{~m} \\
& \mathrm{~m}=3,14208220804
\end{aligned}
$$

d) Se calcula la tasa máxima de producción para el proceso SAGD.

$$
\mathrm{q}=2 \mathrm{~L} \sqrt{\frac{1.3 \mathrm{~kg} \phi \Delta \mathrm{S}_{\mathrm{o}} \mathrm{h}}{\mathrm{m} v_{\mathrm{S}}}}
$$

Antes de emplear la ecuación propuesta, se debe determinar los valores de los parámetros que intervienen en dicha ecuación y convertirlos a un sistema de unidades consistente, en este caso, se empleará el Sistema Internacional - SI.

La permeabilidad tiene que estar en $\mathrm{m}^{2}$. Entonces:

$$
k=1.3 \operatorname{Darcy}\left(\frac{0.9869 \times 10^{-12} \mathrm{~m}^{2}}{1 \text { Darcy }}\right)(0.4)=4.74 \times 10^{-13} \mathrm{~m}^{2}
$$

NOTA: El valor de 0.4 es la permeabilidad relativa promedio estimada. La variable $\alpha$ es la permeabilidad efectiva para el flujo del petróleo

La difusividad térmica del yacimiento debe estar en $\mathrm{m}^{2} / \mathrm{s}$, entonces:

$$
\alpha=\frac{0.05 \mathrm{~m}^{2}}{86400 \mathrm{~s}}=5.79 \times 10^{-7} \frac{\mathrm{m}^{2}}{\mathrm{~s}}
$$

El diferencial de saturación es:

$$
\Delta \mathrm{S}_{\mathrm{o}}=0.75-0.15=0.60
$$

Como se determinó en el paso 1 , el valor de la viscosidad cinemática a $210^{\circ} \mathrm{C}$ es $6.7 \mathrm{cSt}$, este valor transformarlo a $\mathrm{m}^{2} / \mathrm{s}$.

$$
6.7 c S t\left(\frac{10^{-6} \mathrm{~m}^{2} / \mathrm{s}}{1 c S t}\right)=6.7 \times 10^{-6} \frac{\mathrm{m}^{2}}{\mathrm{~s}}
$$

Luego empleando la ecuación lineal de drenaje: 


$$
\begin{gathered}
q=2(400) \sqrt{\frac{1.3\left(4.74 \times 10^{-13}\right)(9.81)\left(5.79 \times 10^{-7}\right)(0.224)(0.60)(30)}{(3.1421)\left(6.7 \times 10^{-6}\right)}} \\
q=6.53 \times 10^{-4} \frac{m^{3}}{s}\left(\frac{86400 s}{1 d^{\prime} a}\right)=56.38 \frac{m^{3}}{d^{\prime} i a}\left(\frac{6.29 b l}{1 m^{3}}\right) \\
q=354.62 \frac{\mathrm{bl}}{\text { día }}
\end{gathered}
$$

Aplicando el modelo matemático empírico propuesto por Roger Butler, se determina que de acuerdo a las características petrofísicas y de yacimiento del Campo Pungarayacu, se produciría una tasa de producción estimada de 354,62 barriles por día bajo el proceso SAGD para un pozo de estas condiciones de reservorio, incluyendo espesor y longitud del mismo.

Por otra parte, respecto a las ventajas y desventajas esperadas en la zona posterior a la aplicación del sistema SAGD, la teoría de Butler en la que se fundamenta el proceso, tiende a producir tasas pesimistas, debido a:

- Ignora el calor llevado al yacimiento por el agua condensada del vapor.

- Ignora el hinchamiento de la zona de petróleo debido a la intrusión del agua condensada del vapor y por la expansión térmica.

- Debido a los efectos capilares, la interfase no es suave tal como lo supone la teoría y por el contrario, es muy irregular a escala de poro. El agua tiende a llenar los poros pequeños y mantener fuera al vapor, mientras que éste entra más fácilmente en los poros más grandes, resultando en una extensión de la transferencia de calor y una mayor tasa de calentamiento. Este efecto no es muy grande para SAGD pues en este proceso la profundidad de la penetración del calor (o sea, el espesor de la capa de petróleo móvil) es grande en comparación con el espesor de zona de transición vapor-líquido.

- Estos efectos tienden a hacer que el área disponible para el flujo del petróleo sea mayor que en la teoría.

- Este método teórico da resultados conservadores respecto a la tasa real de producción que se obtendría mediante el proceso SAGD, asegurando por tanto que la predicción no sea demasiado optimista para los cálculos financieros de la empresa que en su momento se haga cargo de la operación del Campo Pungarayacu.

- Por otro lado, la teoría desprecia el aumento en viscosidad que ocurre a medida que parte del agua condensada entra en el petróleo para formar emulsiones Agua/Petróleo, particularmente cuando las cámaras de vapor se están elevando hacia el tope del yacimiento. 
- La profundidad de la penetración del calor es menor cuando el crudo es de viscosidad baja y la temperatura del vapor, alta, o sea, cuando el petróleo es menos viscoso y drena más rápidamente, produciendo un avance más rápido de la interfase.

\section{Conclusiones y Recomendaciones}

El presente trabajo plantea la necesidad de desarrollar una metodología eficiente que permita determinar los factores de mayor influencia en el proceso SAGD y, a su vez, proporcionar una forma de optimizar ciertos parámetros, con el fin de conseguir la exitosa implementación de éste en un yacimiento; por ello de acuerdo a las características petrofísicas y de yacimiento del Campo Pungarayacu, se calculó que el mismo tendría una tasa de producción de petróleo estimada de 354,62 barriles por día bajo el proceso SAGD, aplicando el modelo matemático empírico propuesto por Roger Butler.

La zona del Campo Pungarayacu en la que se aplicaría el proceso SAGD es la formación Hollín Inferior, por sus características petrofísicas y por su espesor que varía entre 23 y 57 metros.

Los parámetros de yacimiento de mayor impacto en la producción de petróleo durante el proceso SAGD en los yacimientos de crudo pesado, en base a estudios realizados de simulación, son: el espesor de la arena, la porosidad media del yacimiento, la temperatura inicial, la desviación estándar de la porosidad, la relación entre la permeabilidad vertical y horizontal Kv/Kh y el grado API del crudo.

El espesor de la arena, es el parámetro de yacimiento que resulta más influyente para el proceso, con una contribución de 10\% aproximadamente a la variabilidad del petróleo producido. De esta forma, puede esperarse que a mayor espesor de la arena mayor sea la producción de petróleo en el proceso SAGD.EI segundo parámetro de yacimiento de mayor relevancia es la porosidad de la roca reservorio, con una contribución del 9\% aproximadamente a la variabilidad del petróleo producido.

En cuanto a los parámetros operacionales, los más influyentes en el proceso son: la tasa de inyección de vapor con una contribución a la variabilidad del petróleo producido de 16\%; la duración del período de precalentamiento con 3\%; la calidad del vapor inyectado con el $5 \%$ y la distancia vertical entre el pozo inyector y el pozo productor.

Además, este trabajo es de vital relevancia en las áreas de la Ingeniería de Yacimientos y en los métodos de recuperación térmica de petróleo. También, constituye un aporte en la conformación de una base de información que sería de utilidad para proyectos en el área, así como para investigaciones que involucren esta temática.

En la actualidad la aplicabilidad del proceso SAGD a un determinado yacimiento de crudo pesado o extra-pesado, así como los parámetros operacionales usados, son determinados generalmente 
por criterios generados de las experiencias adquiridas en las operaciones de yacimientos similares que han sido drenados por este método. De este modo, si se pretende lograr un rendimiento satisfactorio, es necesario realizar a priori un proceso de selección de los parámetros operacionales óptimos para dicho yacimiento.

\section{Simbología}

\begin{tabular}{|c|c|}
\hline Símbolo & Definición - unidades \\
\hline $\mathrm{T}_{\mathrm{s}}$ & Es la temperatura de saturación del vapor, $\left[{ }^{\circ} \mathrm{C}\right]$ \\
\hline $\mathrm{P}_{\mathrm{S}}$ & Es la presión en la cámara de vapor, [MPa] \\
\hline$v_{\mathrm{S}}$ & Es la viscosidad cinemática del vapor, $\left[\mathrm{m}^{2} / \mathrm{s}\right]$ \\
\hline $\mathrm{m}$ & Es una constante adimensional \\
\hline $\mathrm{b}$ & Es una constante adimensional \\
\hline$\frac{1}{\mathrm{~m} v_{\mathrm{s}}}$ & $\begin{array}{l}\text { Es una expresión que define una integral definida para determinar el valor } \\
\text { adimensional de } m\end{array}$ \\
\hline q & Es la tasa máxima de producción empírica bajo el proceso SAGD, [bl/día], [m³/s] \\
\hline $\mathrm{k}$ & Es la permeabilidad efectiva para el flujo de petróleo, [Darcy], $\left[\mathrm{m}^{2}\right]$ \\
\hline$\alpha$ & Es la difusividad térmica, $\left[\mathrm{m}^{2} / \mathrm{s}\right]$ \\
\hline$\Delta \mathrm{S}_{\mathrm{o}}$ & $\begin{array}{l}\text { Es la saturación de petróleo remanente, saturación actual menos la saturación } \\
\text { residual } \Delta \mathrm{S}_{\mathrm{o}}=\mathrm{S}_{\mathrm{oi}}-\mathrm{S}_{\mathrm{or}} \text {, [fracción] }\end{array}$ \\
\hline $\mathrm{g}$ & Es la aceleración de la gravedad cuyo valor es $9.8 \mathrm{~m} / \mathrm{s}^{2}$ \\
\hline $\mathrm{L}$ & Es la longitud del pozo horizontal perforado en la base del yacimiento, [m] \\
\hline$\phi$ & Es la porosidad, [fracción] \\
\hline $\mathrm{h}$ & $\begin{array}{l}\text { Es el espesor total que es la distancia entre el pozo productor y la base del } \\
\text { yacimiento, [m] }\end{array}$ \\
\hline $\mathrm{S}_{\mathrm{o}}$ & Es la saturación de petróleo, [fracción] \\
\hline
\end{tabular}

\section{Bibliografía}

Alboudwarej y otros (2006). La importancia del petróleo pesado. Oilfield Review Schlumberger's technology journal, 38-40.

Alvarado, D., y Banzer, C. (2002). Recuperación Térmica de Petróleo. Caracas: Adafel Rincón Mora.

Butler, R. (1991). Thermal Recovery of Oil and Bitumen. Alberta: Prentice Hall.

Chekhonin y colaboradores (2012). Cuando las rocas se calientan: Las propiedades térmicas de las rocas yacimiento. Oilfield Review Schlumberger's technology journal, 20.

Fernández, J. (2007). Simulación integrada subsuelo-superficie del proceso SAGD en un modelo estocástico de yacimiento (Tesis de pregrado). Universidad Central de Venezuela. Caracas. 
García, E. (2007). Optimización del Proceso SAGD en un modelo Conceptual de yacimientos utilizando técnicas de Diseño Experimental (Tesis de pregrado). Universidad Central de Venezuela.

Caracas.

Recuperado

de

http://saber.ucv.ve/jspui/bitstream/123456789/2697/1/Tomo.pdf 